International Journal of Engineering \& Technology, $7(2.32)(2018) 427-430$
International Journal of Engineering \& Technology
SPC
Website $:$ www.sciencepubco.com/index.php/IJET
Research paper

\title{
Analytic Method for Estimating the User Behavior Patterns in Multimedia Social Networks
}

\author{
Kesavarapu Kowshik $^{1}$, M V S S Sandeep ${ }^{2}$, Sai Gottipati Mounika ${ }^{3}$, Animesh Adhikari ${ }^{4}$ \\ ${ }^{1,2,3,4}$ Dept of CSE, KLEF, Vaddeswaram, Guntur, INDIA \\ *Corresponding author E-mail: Kesav.koushik@gmail.com
}

\begin{abstract}
Now a days the multimedia social networks plays major role in our daily life. All the earlier MSNs are validated and developed very well. The past decade has witnessed the emergence and progress of multimedia social networks (MSNs), which have explosively and tremendously increased to penetrate every corner of our lives, leisure and work. As well as, the users are enabled by Mobile internet \& terminals for accessing the MSNs where ever they are and when they want with the help of any identity. It may be a group or a role. So, it become very complicated \& comprehensive to provide the behavior's interaction between MSNs as well as in users. The implemented system having the advancements and developed framework of the analytics in a particular domain; which is called as SocialSitu, And We implemented an algorithm which is named as novel for the analysis of the serialized users intention according to the typical GSP which is the short form of Generalized Sequential Pattern. An enormous number of user's behavior records were broken for exploring the usual sequence mode. It is mandatory for guessing the intention of the user. We considered the two types of intentions. Those are playing multimedia \& sharing multimedia. These 2 are widely used in regular MSNs with the help of intention serialization algorithm in control of various min support threshold (Min_Support). With the help of microscopic behavior analysis of the users, we find out the each user behavior patterns which are in optimized manner in control of the Min_Support. Based on the different identities of the user, the behavior patterns of the users may be varied in session data which is very large.
\end{abstract}

Keywords:multimedia social networks, situation analytics, intention prediction, behavior pattern, big data.

\section{Introduction}

Now a days the we can observe the growth of the users and contents in multimedia as there are rapid advancements takes place. With the help of large scale video data set \& MSNs, the digital contents are easily accessed by the users. As a result, the communication between user \& user and user \& system increases. Hence, providing of personalized services timely and rapidly may become complex interaction. It became a challenge in social networks of the multimedia. Usually the computing \& speaking of the multimedia can be classified into three types. First one

is data centric compression of multimedia, communication of the content centric multimedia \& multimedia. The analysis of the user centric social media considers the trust modeling of the user and mining paths propagation \& sharing of the digital right ad forensic which is digital in nature. So that, content users of the multimedia exact needs understanding \& guessing in various situations study is not done well. The first proposal is CA which is the short form of Context-Aware. Here, the context is defined in terms of location, nearby people, objects, objects changes all these are considered a set. After that the Situ theory proposed by Chang with the combination of environment of the service using the awareness of the situation for regulating the changing updates our run time service developments. Hence, we reached the user needs which changes and we can offer personalized user services. The awareness about the situation is necessary for offering the timely response and good environment in service. then we can easily adapt to the dynamic service. The human is considered as open and complex system in social media networks. The intention of one person may change at any time so that the needs of the user also changed. However, as the behavior of the human and context is dynamic in nature. The needs of user may changes based on the dynamic change characteristics. we can say that based on various studies. According to the user situation and resources feedback the user intention can be reflected. According to the intention of the user, the user personalized services timely can be done by a system. It leads the experience of the user services. There are various roles in various group for the users in social media networks. The intention of the user may be changed based on the various identifications. The user's behavior changes are reflected by the intention changes of the user. Coming to the social media networks, based on the various identities, user intention analysis may not reach the full accuracy by Situ theory. The primary motivation of the social media networks sequences mode user intention analysis. The major advantages of this implemented system are enrichment as well as advancement of the Situ theory in social domain. and second one is proposal of the novel algorithm to analyses the behavior pattern of the user. The main motto of this implemented system is prediction of the in depth user intention and earlier work large volume according to the mental intention.

\section{Related work}

Situation analysis theory importance and influence as well as Situ theory in software engineering studied. The details of the situ system that gives services of the personal, based on detection of 
the service update in real time. Ming et already raised the analysis of the spatial method according to the theory of Situ. After that the Rahman et al proposed the sharing of the user data in social circle with friends with the help of a service which is social. SenseFacen framework used for user's service recommendation with information of multimedia and network of user sensor of the data. Shen et al stated that the relationship of the social network and environment of the surroundings. It can able to detect the situation of the user and preferences, relationship of the users which are nearer by computing the situation of the user similarities $\&$ guessing the user situation. After that Zhang et al, advanced $\mathrm{N}$ gram prediction algorithm for predicting the web access request which come in future with log data of the server. Bar-David etal proposed guessing the user location based on the earlier objects that are in moving. If we discard the moving object errors. According to the different context positions for predicting the vehicle position, context-aware position prediction algorithm proposed. Lee-et-al designed another algorithm to allow the smart phones users for accessing the easy services in time. For predicting the intention of the user and offering the correct services the condition of the event behavior model along with rule induction algorithm stated. It is used for finding the mobile users intentions MSNs. a data of the user noise as well as discrete in MSN. For mining and analysis this will not be useful. So that we have to gathered the date of the user preprocess earlier. The theory of the change situation analytics is for the software which is not much appropriate for engineering applications. For providing the best services personally, with context and MSN situations. For gathering the sequence of the intention of the user I proposed the algorithm of the novel to analyses the user sequences of SocialSitu(t) with GSP advancements.

\section{MSNs SITU Framework Extension}

There will be huge number of users in various groups in MSNs. They are playing different role in those groups. So the desires of the persons may deepen on the role. The enrichment of the Situ advancement of this framework in social media, represents in the following section

Definition 1 (Situation(it)): At t time the situation can be represented It represents the that having the 3ple, Situation $(t)=$ $\{d, A, E\}$.

Here the user desire referred by $d$ at $t$ interval, user action can be represented by $A$. $d$ is the achievements. At time t, the context of the environment referred by the. $E$

Definition 2 (SocialSitu(t)): Situation can be represented by it at

t. It is the Situation(t) extensional in the domain which is social in nature. SocialSitu(t is the 4thple SocialSitu(t) = $\{I D, d, A, E\}$.

Identity information of the user can be represented by the $I D$; At $t$,

\section{The desire of user can be represented by $d$}

Definition 3 (ID): Identity information of the user can be represented by the $I D ; 2$ ple $I D=\{$ Group, Role $\}$.

There should be good relation between the group of the user and role in that group in MSNs. .

Definition 4 (Group): For a specific reason, sometimes small group are created in social media. It is also a part of the network of the social mediIt's a part of the whole social media network.

Definition $5($ Role, $R)$ : The role of the user in MSNs. set $R=\{r 1, r 2, \ldots, r n\}$,
Definition 6 (Desire, $D$ ): While utilizing the social media, it is the thing what a user need and get

$\{d 1, d 2, \ldots, d n\}, \quad d i(1 \leq i \leq n)$ are referred to desire of the user at $i$.

Definition 7 (Goal, $G$ ): common target of the user's $G=\{g 1, g 2$, $\ldots, g n\}$

Definition 8 (Intention, I): It represents the

SocialSitu( $t$ user sequence towards the achieve target from the point to

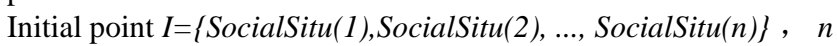
$\in N$, SocialSitu(1)

The target can be achieved by the user with the help of intention sequence

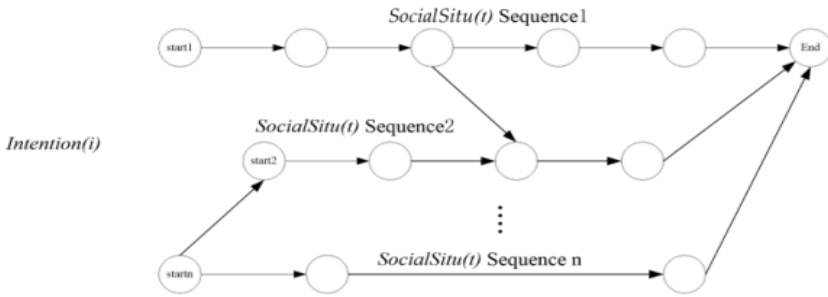

Fig.1. Intention sequence here, at a particular point SocialSitu(t) represented by every point.

$\operatorname{startj}(1 \leq \mathrm{j} \leq \mathrm{n}, \mathrm{j} \in \mathrm{N})$

Intention(i). we may or may not have same are different initial points.

End represents the closing point Intention (i).. at Intention(i) every sequence, same node may be terminated except the end point.

\section{User Intention Serialization Algorithms}

The SocialSitu(t) which is frequent at specific goal. The intention of the user sequence is presented in historical access record in MSNs. The database saves the sequence of the user intention along with a goal which is specific. By comparing the current user sequence and intention user sequence, we can guess the present user intention for making the perfect reply for the request of the user. Then we can provide the service which is personalized. Finding of the intention of the user is the major issues here.

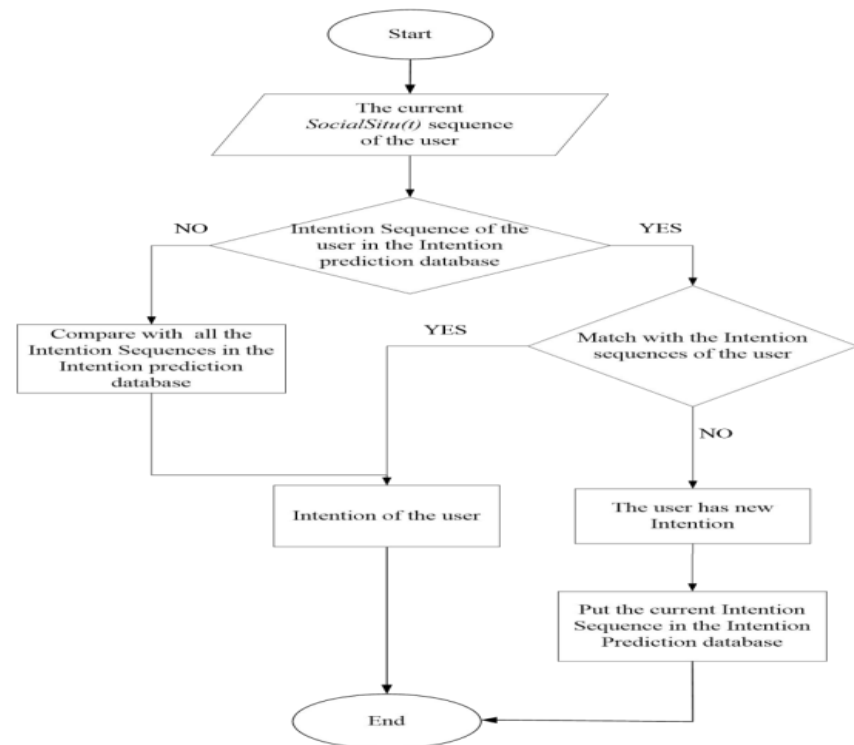

Fig.2. user flowchart Intention prediction 
For finding the relationship among the different items this rule which is named as association rule in big. transaction set can be represented by DS. here we have the term "item" which is nothing but attributes. . data item set, $I=\{i 1, i 2, \ldots$, $i m$,

$|I|=\mathrm{m}$,

$A \Rightarrow B$

,i $\in N, i \geq 1$.

$A \subseteq I, B \subseteq I$

$\& A \cap B=\Phi$

of the association rule; $A \cup B$

Corresponds to this rule the item set which All frequent SocialSi$t u(t)$ related to a certain goal achievement in a user's historical access record consists:

- Support: item set number R presented in DS named as R supporting number, rule

$R \Rightarrow S$

Represents the number in the RMSNs item sets. Must have the minimum single intention sequence. The sequence of the user's intention along with a particular specific goal which the database can save. Comparison of the current user sequence \& intention user sequences in database for guessing the present user intention. $R \Rightarrow S$ is t $(R \Rightarrow S)=\mathrm{P}(R \cup S)$.

- Confidence: Rule Confidence $R \Rightarrow S$ represents probability where complete data set DS having A includes B ( $R \Rightarrow$ $S)=\mathrm{P}(\mathrm{R} \mid \mathrm{S})$.

Min_Support - tem set which is frequent. satisfies the Min_Support \& (Min_Conf)

Intention $(i)$

serialization done according to the rule for detecting every sequence for respective intent at every ending point.

Intention(i) sequence as taken as association result rule, this helpful for obtaining the association antecedent rule. The algorithm of the Intention serialization represents in Algorithm 1 intention serialization algorithm flowchart described in Fig.3. serialization algorithm steps according to rule of the association as below:

(1) The database of the web log database can be scanned later processing the data in definition 7 can be identified association rule result for generating the $L k+1$ sequence $\mathrm{k}+1$ length,

(4) 3rd Step repeats till where no longer new person sequence can be generated. sequences of the SocialSitu(t) in relation to Intention $(i)$ target $g$ ' obtained.

(5) All sequences of the SocialSitu(t) sequences respected to database of the target like a user end point in Intention( $i)$,

$' i+1$ obtained and saved as Intention $(i+1)$. Then, recorded like $G^{\prime}=\left\{g^{\prime}, g^{\prime}, g^{\prime}\right\}, 1 \leq m \leq n, G^{\prime} \subset G$.

repeat the (2), (3), and (4). steps.

(2) From step (1) g getting utilized as association result rule. every SocialSitu(t) utilized association rule antecedent for computing support each rule \& find a rule that satisfy Min_Support.

(3) The rule antecedents came with step (2)

(4) utilized for setting set $L 1, L k$ candidate sequence generation is $C k+1 \mathrm{k}+1$ length.

(5)DS scanned data set, compute with sequence of each candidate like antecedent $\& g$
(6) till no new goal. Link operation: After s1 obtained the sequence equal to $\mathrm{S} 2$. Where the $\mathrm{S} 2$ added to $\mathrm{S} 1$.

Pruning operation: when particular candidates sub-sequence pattern is not equal to this candidate sequence pattern. Hence it can be deleted.

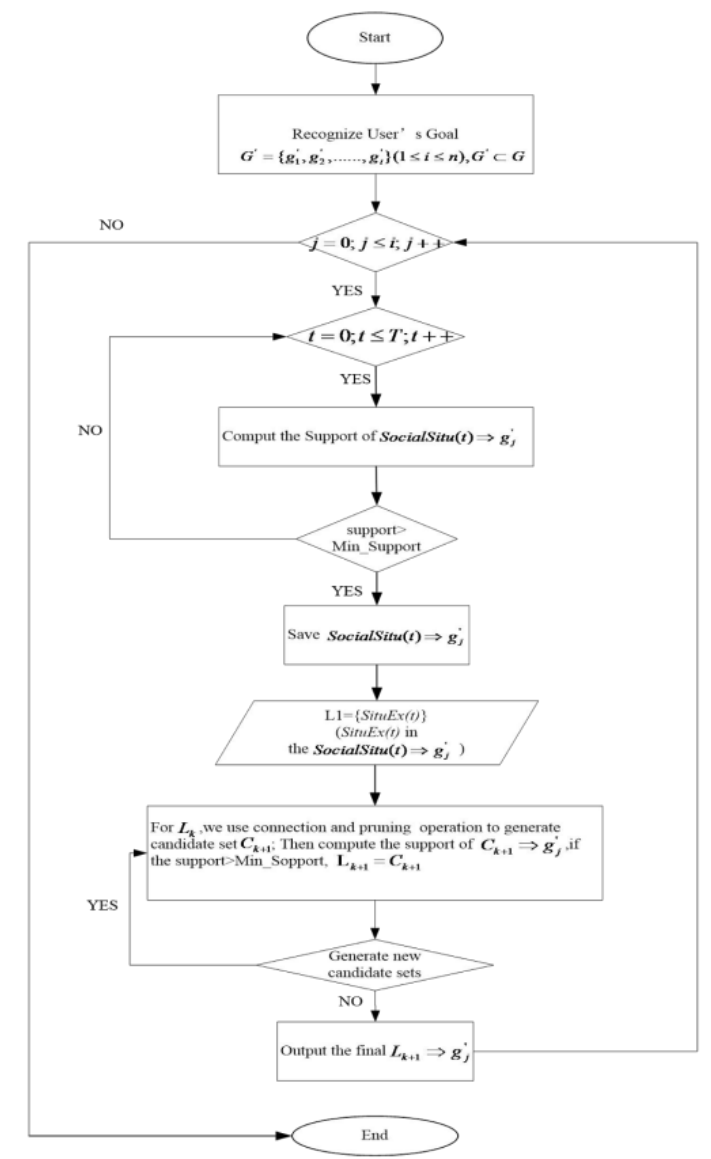

Fig.3. Algorithm of situational aware intention serialization algorithm Flowchart

\section{Conclusion}

The awareness of situations become mandatory in typical MSNs environment. Environment of the User, behavior can be changing as well as the changing of the intention also may see in individual's. for adopting the user changes identities which are dynamic in nature and social domain. Here, we have the Si which is the extended and enriched. SocialSitu theory is built for networks of the social media. serialization algorithm achieved and implemented for social networks which runs on multimedia. The sequence Moe of the user intention which is frequent can be obtained with this algorithm. If the identity of the user changed, we can know the different ID which describes behavior pattern, confidently we obtained the various sequences of the SocialSitu(t), on same intention if his or her group or role can be changed. Coming to the works which can be done in future, we can guess the various in depth intentions of the user buy the help of typical user cloud sequence patterns of the intentions. So that we can use the SocialSitu. Implemented system is for improving the multimedia system recommendations as well as MSNs killer applications. .

\section{References}

[1] "Y. G. Jiang and J. J. Wang, "Partial Copy Detection in Videos: A Benchmark and an Evaluation of PopularMethods," IEEE Trans. Big Data, vol. 2, no. 1, pp. 32-42, Jan/Mar 2016, doi:10.1109/TBDATA.2016.2530714." 
[2] C. K. Chang, "Situation Analytics-A foundation for a new software engineering paradigm," Computer, vol. 49, no. 1, pp.24-33, Jan. 2016.

[3] P. Cui, W. Zhu, T. S. Chua, and R. Jain, "Social-Sensed Multimedia Computing," IEEE Multimedia, vol. 23, no. 1, pp. 92-96, Jan/Mar 2016

[4] X. L. Shen, M. K. O. Lee, and C. M. K. Cheung, "Exploring online social behavior in crowdsourcing communities: A relation-ship management perspective," Comput. Hum. Behav., vol. 40, pp. 144 151, Nov. 2014

[5] C. Tekin and M. Van Der Schaar, "Contextual online learning for multimedia content aggregation," IEEE Trans. Multimedia, vol.17, no.4, pp.549-561,Apr.2015, doi:10.1109/TMM.2015.2403234. Networks," Soc. Netw. Anal. Min., vol. 3, no. 4, pp. 969-979, Dec.2013.

[6] D. C. A. Bulterman, P. Cesar, and R. L. Guimaraes, "Sociallyaware multimedia authoring: Past, present, and future," ACM Trans. Multimedia Comput. Commun. Appl., vol. 9, no. 1 SUPPL,Oct. 2013, doi: $10.1145 / 2491893$

[7] H. Ming, C. K. Chang, and J. Yang, "Dimensional SituationAnalytics : from Data to Wisdom," Proc Int Comput Software Appl Conf, vol. 1, pp. 50-59, Jul. 2015, doi:10.1109/COMPSAC.2015.199.

[8] Azfar, K.-K. R. Choo, and L. Liu, "Forensic Taxonomy of Android Social Apps," J. Forensic Sci., preprint, Nov. 2016, doi: 10.1111/1556-4029.13267.

[9] D. Quick and K.-K. R. Choo, "Big forensic data management in heterogeneous distributed systems: quick analysis of multimedia forensic data," Softw. Pract. Exper., preprint, 2016, doi 10.1002/spe.2429. [10

[10] P. Cui, W. Zhu, T. S. Chua, and R. Jain, "Social-Sensed Multimedia Computing," IEEE Multimedia, vol. 23, no. 1, pp. 92-96, Jan/Mar 2016.

[11] B. N. Schilit and M. M. Theimer, "Disseminating Active Map Information to Mobile Hosts," IEEE Network, vol. 8, no. 5, pp. 22-32, Sep/Oct 1994.

[12] K. Srinivasan, P. Agrawal, R. Arya, N. Akhtar, D. Pengoria, and T. A. Gonsalves, "Context-aware, QoE-driven adaptation of multimedia services," 5th International Conference on Mobile Wire-less Middleware, Operating Systems, and Applications, pp. 236-249, Nov. 2012, doi: 10.1007/978-3-642-36660-4_17.

[13] C. Tekin and M. Van Der Schaar, "Contextual online learning for multimedia content aggregation," IEEE Trans. Multimedia, vol. 17, no. 4, pp. 549-561, Apr. 2015, doi: 10.1109/TMM.2015.2403234.

[14] D. C. A. Bulterman, P. Cesar, and R. L. Guimaraes, "Sociallyaware multimedia authoring: Past, present, and future," ACM Trans. Multimedia Comput. Commun. Appl., vol. 9, no. 1 SUPPL, Oct. 2013, doi: 10.1145/2491893.

[15] C. K. Chang, H. Y. Jiang, H. Ming, and K. Oyama, "Situ: A situation-theoretic approach to context-aware service evolution," IEEE Trans. Serv. Comput., vol. 2, no. 3, pp. 261-275, 2009, doi: 10.1109/TSC.2009.21 